\title{
Increased anthropogenic activities destabilized bacterial co-occurrence networks in a subtropical river
}

\author{
Lemian Liu ${ }^{1}$, Shanshan Wang ${ }^{1}$, and Jianfeng Chen ${ }^{1}$ \\ ${ }^{1}$ Fuzhou University
}

August 13, 2020

\begin{abstract}
Previous studies suggested that in an ecological co-occurrence network with a large proportion of strongly positive correlations, the network members may respond synchronously to environmental changes (e.g synchronously become extinct), resulting in instability of network. Anthropogenic activities have strong influences on the microbial community composition and diversity in river ecosystems, but how their influences on the stability of microbial co-occurrence networks remain unclear. In this study, we used nutrient concentrations (nitrogen and phosphorus) as an indicator for anthropogenic actives, and explored the effects of anthropogenic activities on the stability of bacterial co-occurrence networks in a subtropical river, Xiyuan River. The nutrient concentrations were higher in midstream and downstream areas than in upstream area of Xiyuan River. The proportion and correlations coefficients of positive correlations for bacterial co-occurrence networks strongly and positively correlated with the nutrient concentrations, indicating increased anthropogenic activities destabilized the networks in midstream and downstream areas. To further explore the mechanisms, we found that the changes of network stability were associated with the changes of bacterial functions. Anthropogenic activity tolerant bacteria (e.g. nutrient removal, aromatic degradation and pathogen bacteria) and their linked bacterial members formed a largely and strongly positive module in the midstream and downstream networks, therefore destabilized the networks. Based on network perspective, our results provide new insight in the mechanisms of anthropogenic activities alter riverine microbial communities.
\end{abstract}

\section{Hosted file}

MS XYJ 20200812.doc available at https://authorea.com/users/350568/articles/475391-increasedanthropogenic-activities-destabilized-bacterial-co-occurrence-networks-in-a-subtropicalriver 